\title{
OS CROCODILIFORMES DA FORMAÇÃO MARÍLIA (BACIA BAURU, CRETÁCEO SUPERIOR) NA REGIÃO DE MONTE ALTO, ESTADO DE SÃO PAULO, BRASIL
}

\author{
FABIANO VIDOI IORI \\ Museu de Paleontologia "Prof. Antonio Celso de Arruda Campos", Praça do Centenário, \\ Centro de Artes, Monte Alto, SP, Brasil.biano.iori@gmail.com \\ $\dagger$ ANTONIO CELSO DE ARRUDA CAMPOS \\ Museu de Paleontologia "Prof. Antonio Celso de Arruda Campos", Praça do Centenário, \\ Centro de Artes, Monte Alto, SP, Brasil.
}

\begin{abstract}
THE CROCODYLIFORMS FROM THE MARÍLIA FORMATION (BAURU BASIN, UPPER CRETACEOUS) IN THE MONTEALTO REGION, SÃO PAULO STATE, BRAZIL. This study presents the oldest historical record of fossil crocodyliforms in the region of the Monte Alto, SP, besides the analysis of an additional fossil from the same site. The specimens come from the Marília Formation and they are the only known local occurrences of Crocodyliformes cranial fragments in this stratigraphic unit. This study expands the knowledge about Crocodyliformes of the Bauru Basin and contributes to the definition of the regional paleofauna during the Maastrichtian.
\end{abstract}

Key words: Crocodyliformes, Bauru Basin, Marília Formation.

\section{INTRODUÇÃO}

Os trabalhos sistemáticos de prospecções paleontológicas realizados em sedimentos cretácicos do Grupo Bauru no Município de Monte Alto (Figura 1), Estado de São Paulo, iniciaram-se na década de 1980 e revelaram uma fauna cretácica composta por biválvios, testudinos, escamados, crocodiliformes, além de dinossauros saurópodes e terópodes (Santucci \& Bertini, 2001; Bertini et al., 2001; Carvalho et al., 2007; Andrade \& Bertini, 2008; Pinheiro et al., 2008; Fachini \& Iori, 2009; Iori \& Carvalho, 2009, 2011; Santucci \& Arruda-Campos, 2011; Ghilardi et al., 2011; Iori \& Garcia, 2012; Tavares et al., 2014; Méndez et al., 2014).

Crocodyliformes é o grupo mais amplamente estudado, com vários espécimes conhecidos e seis espécies descritas para a região: os notossúquios avançados Morrinhosuchus luziae Iori \& Carvalho, 2009, Caipirasuchus montealtensis (Andrade \& Bertini, 2008) e C. paulistanus Iori \& Carvalho, 2011; o baurussuquídeo Stratiotosuchus maxhechti Campos, Suarez, Riff \& Kellner, 2001; o peirossaurídeo Montealtosuchus arrudacamposi Carvalho, Vasconcellos \& Tavares, 2007; e Barreirosuchus franciscoi Iori \& Garcia, 2012, associado aos trematochampsídeos (Carvalho et al., 2007; Andrade \& Bertini, 2008; Pinheiro et al., 2008; Iori \& Carvalho, 2009, 2011; Iori \& Garcia, 2012).

A presença de crocodiliformes em Monte Alto foi relatada pela primeira vez por Moraes-Rego (1935), referindo-se a uma forma análoga ao gênero Pholidosaurus descoberta por Mathias Gonçalves de Oliveira Roxo em materiais oriundos da região. Contudo, parece ter havido um engano por parte de Moraes Rego quanto à procedência do material, conforme apontado por Price (1950), pois em Roxo (1929) somente foram feitas menções à presença de dinossauros de grande porte em Monte Alto.

Nos anos 1950, uma equipe de funcionários municipais, coordenada por José Pierre, deparou com material fóssil durante um trabalho de remoção de rochas na estrada de acesso ao bairro rural de Água Limpa. Diante da descoberta, entraram em contato com Isidoro Nunes, que fotografou o espécime. O trabalho de retirada de rochas continuou, o barranco foi desmontado e o destino do fóssil não é conhecido.

Em 1974, o paleontólogo Rafael Gióia Martins Neto descobriu na fazenda de propriedade de Sérgio Fuzikawa, grande parte de um crânio articulado à mandíbula (Figura $2 \mathrm{~F}$ ), o qual foi encaminhado para a Universidade Estadual Paulista (UNESP) de Rio Claro (Pinheiro et al., 2008). No estudo de Pinheiro et al. (2008) o material é atribuído a Stratiotosuchus maxhechti, contudo Montefeltro et al. (2011) observaram nesse espécime caracteres distintos dos que ocorrem no holótipo da espécie. Este material constitui a única ocorrência de um baurussuquídeo na região até o momento.

Na década de 1980, Isidoro Nunes procurou um dos autores (ACAC), doou a foto que fez nos anos 1950 e apresentou o local do achado, nas margens da estrada de acesso ao bairro rural da Água Limpa (Figura 1, Sítio 1). Em 1987, as prospecções feitas na área do afloramento resultaram na descoberta de um fragmento craniano. Ainda naquele ano, dois dentes isolados associados a restos de titanossauros foram descobertos no sítio de escavação próximo ao Clube de Campo "Campestre" (Figura 1, Sítio 2). Iori et al. (2009b) apontaram o aspecto obtuso da coroa dentária de um dos dentes (Figura 2A) e sua similaridade com as que ocorrem em Itasuchus.

O agricultor Nelson Gonzáles, em 1990, descobriu crânio e mandíbula de um notossúquio (Figura 2G) no Sítio São José de sua propriedade (Figura 1, Sítio 3). Bertini \& Arruda-Campos (1995) fizeram uma breve descrição do 
material e a associação à família Uruguaysuchidae. Andrade \& Bertini (2008) descreveram detalhadamente o espécime e definiram um novo esfagessaurídeo, cuja espécie foi realocada taxonomicamente por Iori et al. (2013) e renomeada Caipirasuchus montealtensis.

Em setembro de 1995, o estudante Juliano Vidoi Iori descobriu um bloco com vários osteodermos na margem da estrada municipal que liga os municípios de Monte Alto e Taiaçu (Figura 1, Sítio 4). Deste local, em uma área inferior a $4 \mathrm{~m}^{2}$, foram extraídos restos de vários indivíduos, porém somente em maio de 2004 foi removido o holótipo do peirossaurídeo Montealtosuchus arrudacamposi (Figura 2E).

Em um afloramento nas proximidades do distrito de Ibitirama denominado "Sítio Paleontológico do Tonhão" (Figura 1, Sítio 5), foram realizadas várias escavações e coletas durante o ano de 1996. Alguns anos depois, Cledinei Aparecido Francisco identifica parte de uma mandíbula (Figura 2C) dentre os fósseis daquele sítio. Iori et al. (2009a) descreveram o fragmento de dentário (com três alvéolos integralmente preservados, com diâmetros $16 \mathrm{~mm}, 18 \mathrm{~mm}$ e $22 \mathrm{~mm}$ ) e consideraram que o indivíduo figuraria entre os maiores crocodiliformes da Bacia Bauru.

Durante as escavações para a remoção do holótipo de Aeolosaurus maximus Santucci \& Arruda-Campos, 2011, realizadas em 1997 e 1998 na Fazenda Santa Irene (Figura 1, Sítio 6), oito dentes isolados de crocodiliformes foram encontrados associados ao esqueleto de saurópode. Tavares et al. (2010) analisaram tais dentes e os dividiram em três morfotipos, dois destes (Figura 2B) foram associados ao gênero Itasuchus.

Três indivíduos (Figuras 2D, H-I) descobertos por um dos autores (FVI) em 2000 e 2001 embasaram a definição de Morrinhosuchus luziae, Caipirasuchus paulistanus e Barreirosuchus franciscoi, descobertos, respectivamente, nas proximidades do Morrinho de Santa Luzia, na Fazenda São Francisco e no bairro rural do Barreiro (Figura 1, Sítios 7-9). Além destes, um quarto espécime, um Caipirasuchus do Morrinho de Santa Luzia encontrado na mesma época, foi utilizado na descrição do pós-crânio do gênero e figurado por Iori et al. (2016).

Em 2007, o agricultor Sérgio Luiz Frare descobre na Fazenda Santa Irene, um espécime de Morrinhosuchus luziae, com crânio e mandíbula quase completos. Iori et al. (2015) divulgaram a ocorrência e apresentaram sucintamente novas informações sobre a morfologia craniana da espécie.

$\mathrm{Na}$ região de Monte Alto ocorrem duas unidades estratigráficas, as formações Adamantina e Marília. A assembleia fossilífera da Formação Adamantina apresenta maior expressividade numérica e taxonômica. Todas as espécies de crocodiliformes identificadas na região reportam a esta unidade e o conhecimento acerca dos táxons da Formação Marília limita-se a um único estudo, onde Iori et al. (2009b) relata, de maneira sucinta, as quatro únicas ocorrências de crocodiliformes na formação, os dois dentes isolados

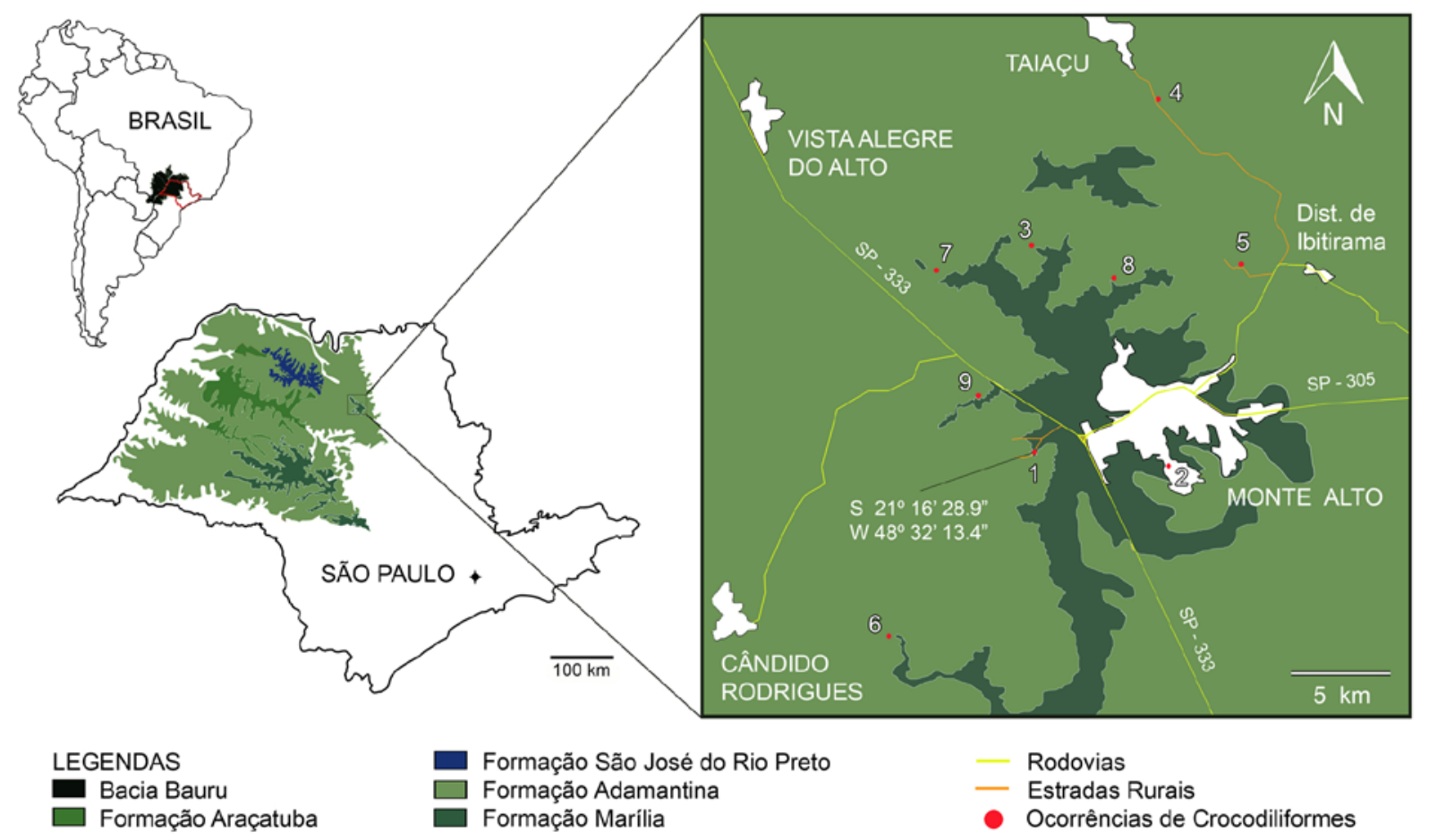

Figura 1. Grupo Bauru, no Estado de São Paulo (modificado de lori et al., 2016). No detalhe, região de Monte Alto com os sítios paleontológicos com ocorrências de Crocodyliformes: Água Limpa (1), Campestre (2), Sítio São José (3), Taiaçu/Monte Alto (4), Tonhão (5), Fazenda Santa Irene (6), Morrinho de Santa Luzia (7), Fazenda São Francisco (8), Barreiro (9).

Figure 1. The Bauru Group in State of São Paulo (modified from lori et al., 2016). In detail, Monte Alto region with paleontological sites and the occurrences of Crocodyliformes: Água Limpa (1), Campestre (2), Sítio São José (3), Taiaçu/Monte Alto (4), Tonhão (5), Fazenda Santa Irene (6), Morrinho de Santa Luzia (7), Fazenda São Francisco (8), Barreiro (9). 

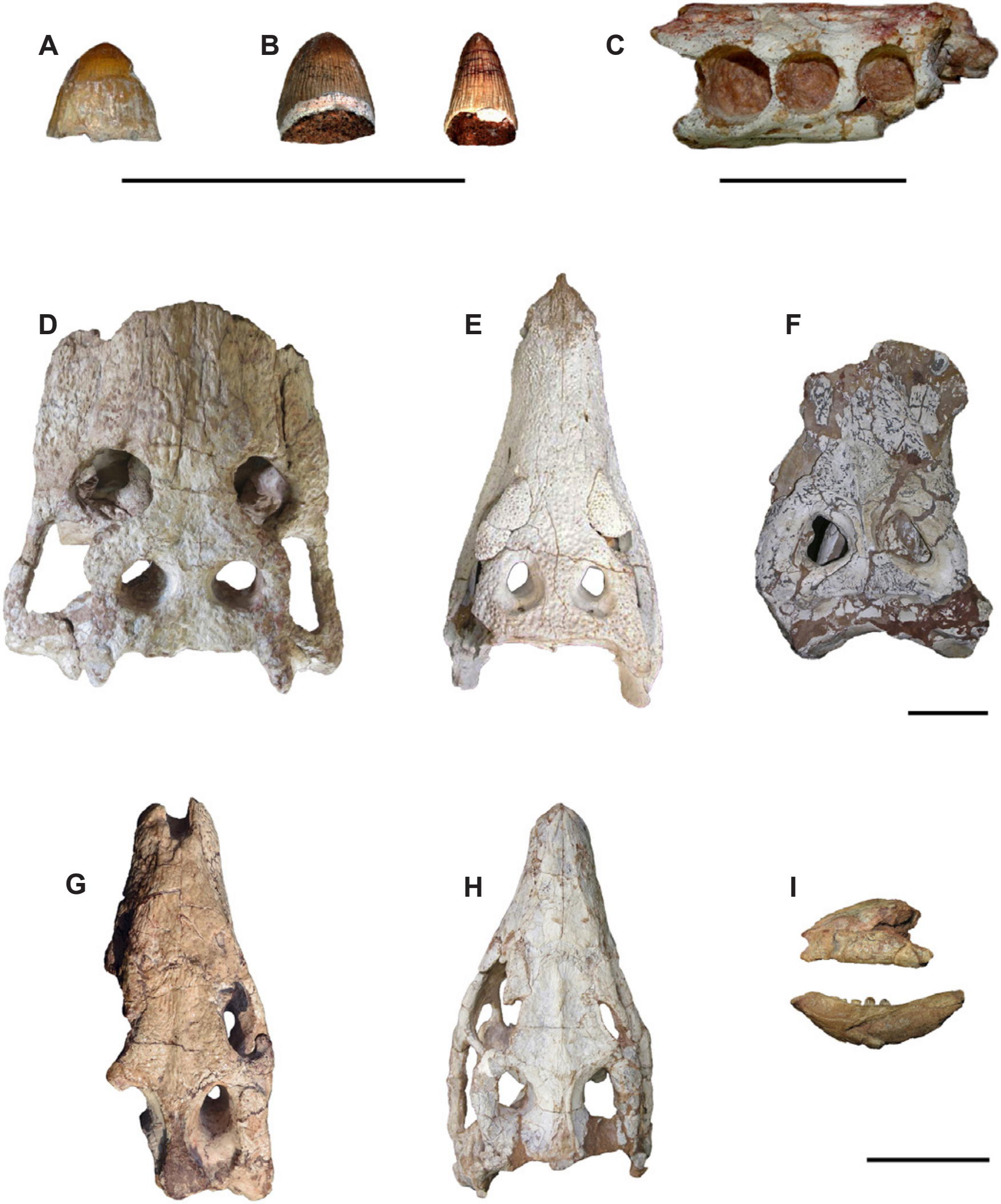

Figura 2. Fósseis de Crocodyliformes da região de Monte Alto. A-B, dentes isolados; C, fragmento de dentário associados ao gênero Itasuchus; D-H, crânios em vista dorsal de Barreirosuchus (D), Montealtosuchus (E), Stratiotosuchus (F), Caipirasuchus montealtensis (G) e Caipirasuchus paulistanus (H); I, fragmentos de crânio e mandíbula de Morrinhosuchus em vista lateral esquerda. Escalas $=5 \mathrm{~cm}$.

Figure 2. Crocodyliformes from Monte Alto region. A-B, isolated teeth; C, fragment of dentary associated to the genus Itasuchus; D-H, skulls in dorsal view: Barreirosuchus (D) Montealtosuchus (E) Stratiotosuchus (F) Caipirasuchus montealtensis (G) and Caipirasuchus paulistanus (H); I, fragments of skull and mandible of Morrinhosuchus in left side view. Scale bars $=5 \mathrm{~cm}$. 
do "Sítio do Campestre", além da fotografia e fragmento craniano (MPMA 02-0005/87) do "Sítio de Água Limpa". Ampliamos aqui a análise destes dois últimos registros inferindo observações taxonômicas e morfológicas dos espécimes, o que permite ampliar o conhecimento acerca da paleofauna da Formação Marília na região de Monte Alto e dos crocodiliformes da Bacia Bauru.

\section{CONTEXTO GEOLÓGICO}

Os fósseis da região de Monte Alto são oriundos da Bacia Bauru (Figura 1), uma ampla depressão cratônica desenvolvida durante o Neocretáceo na porção sudeste da Placa sul-americana (Fernandes \& Coimbra, 1996). O exemplar MPMA 02-0005/87 provém de arenitos da Formação Marília (Membro Echaporã) do Município de Monte Alto, Estado de São Paulo, coordenadas 2116'28.9'S, 4832'13.4"O.

A Formação Marília ocorre exclusivamente em superfície. Seu limite superior é marcado pela superfície topográfica atual e sobrepõe-se à Formação Adamantina por contato interdigitado a abrupto (Paula \& Silva et al., 2003). É composta por bancos métricos de arenitos grossos a conglomeráticos, esbranquiçados, com grãos angulosos, seixos subarredondados de quartzo ou de concreções calcárias, interacamadados com bancos de arenitos grossos maciços com abundantes nódulos e concreções calcárias, raramente ocorrendo intercalações de lentes de lamito e finas camadas de calcário (Dias Brito et al., 2001). A formação é composta por três membros: Serra da Galga, Ponte Alta e Echaporã, todas ocorrendo no Triângulo Mineiro, MG, contudo este último é o único presente no Estado de São Paulo. O Membro Echaporã ocorre nas margens leste e norte da Bacia Bauru, sustenta planaltos escarpados e digitiformes em regiões como as de Marília, Echaporã, Monte Alto e Campina Verde, MG (Fernandes, 2004).

Dias Brito et al. (2001) sugerem que a sedimentação desta unidade teria ocorrido durante o Maastrichtiano, e resultou de aportes episódicos de fluxos de lama (detritos siliciclásticos, fragmentos calcários e soluções calcárias) em pequenas lagoas efêmeras, hidroquimicamente instáveis. Dal' Bó et al. (2009) apontam inúmeros depósitos de paleossolos desta unidade e propõem eventos alternados de aporte eólico e formação de solo. Batezelli (2015) relacionou a taxa de sedimentação e o espaço de acomodação na bacia durante o Maastrichtiano, o aumento do aporte de sedimentos resulta nos acúmulos de depósitos aluviais. A suposição da evolução do clima árido é reforçada pelos indícios de depósitos aluviais dominada

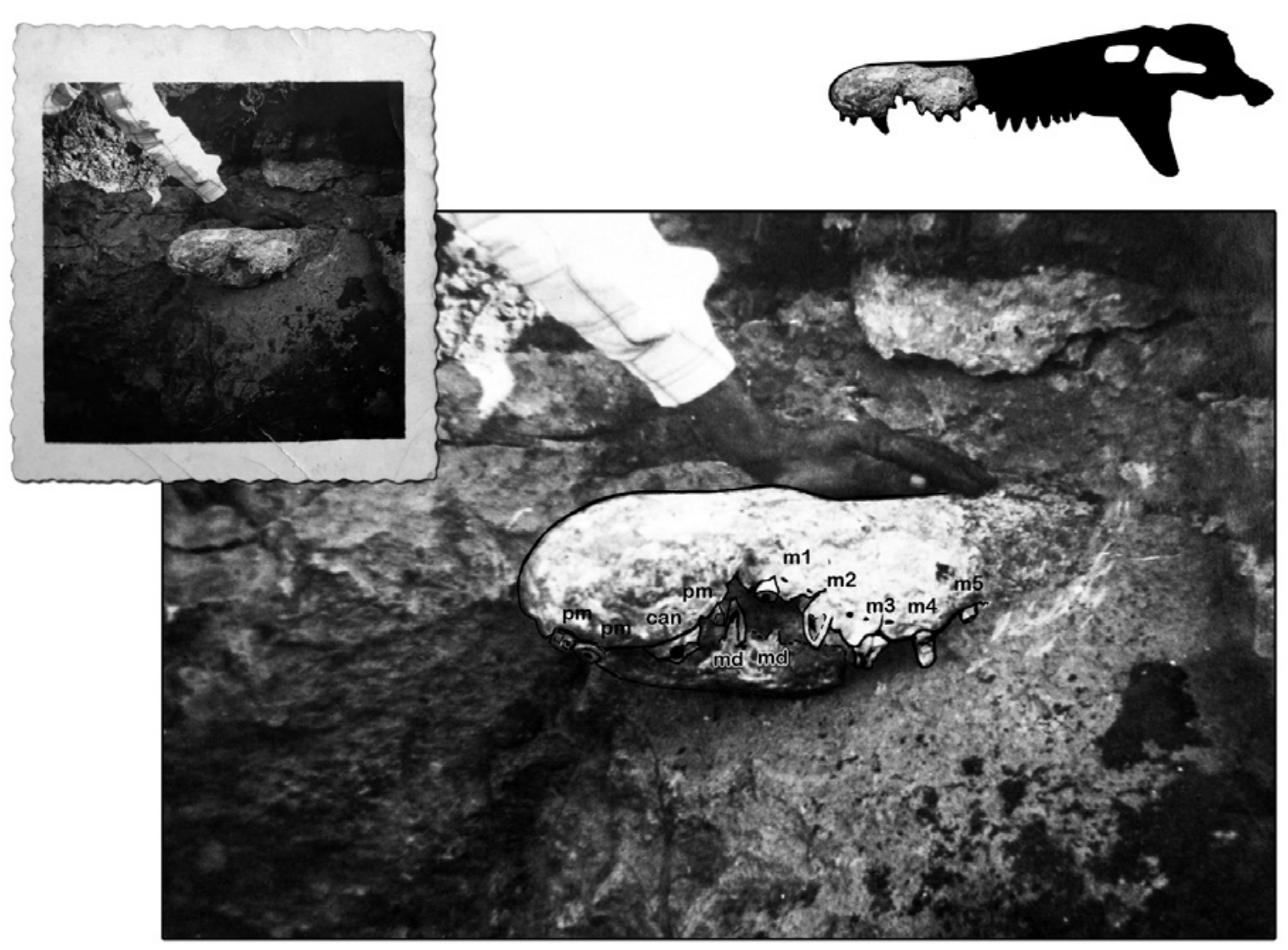

Figura 3. Fotografia da década de 1950 mostrando o fóssil de um crocodiliforme descoberto na região de Monte Alto. No detalhe, as estruturas anatômicas observadas. Abreviações: can, dente caniniforme; m, dentes maxilares; $\mathbf{m d}$, dentes mandibulares; pm, dentes pré-maxilares.

Figure 3. Photography of the 1950s showing the crocodyliform fossil discovered in the Monte Alto region and anatomical structures observed. Abbreviations: can, caniniform tooth; $\mathbf{m}$, maxillary teeth; $\mathbf{m d}$, mandibular teeth; pm, premaxillary teeth. 

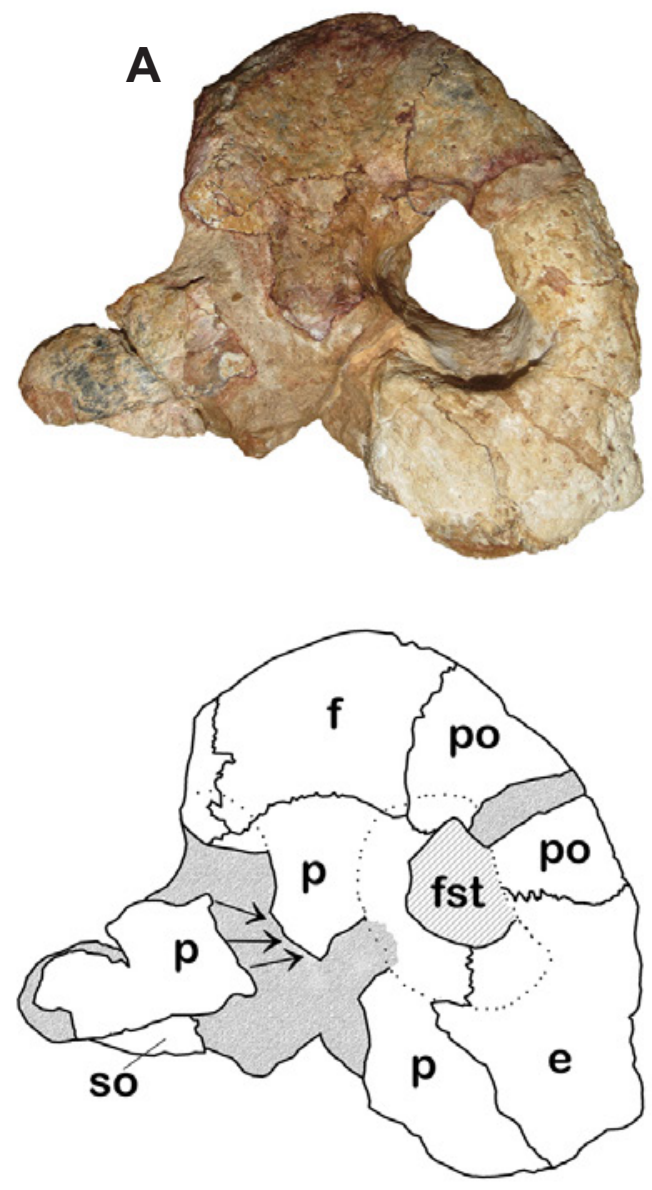

C
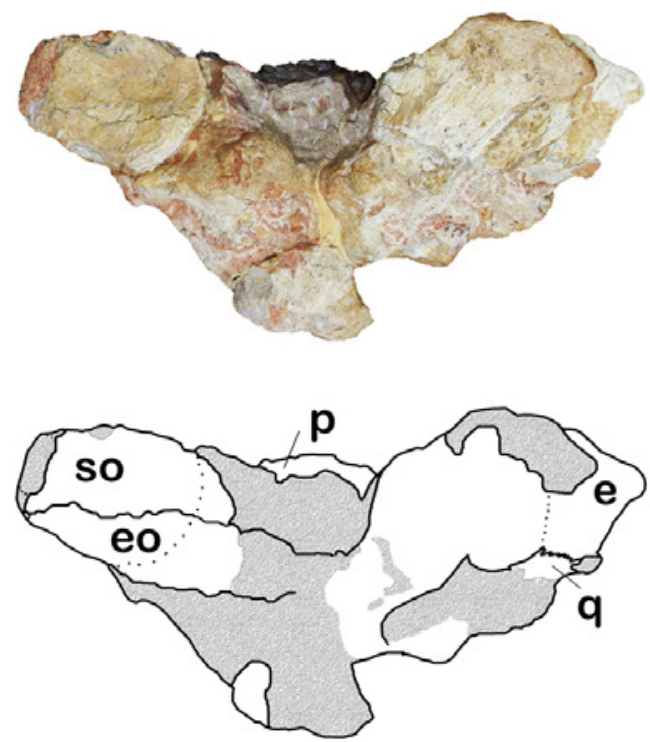
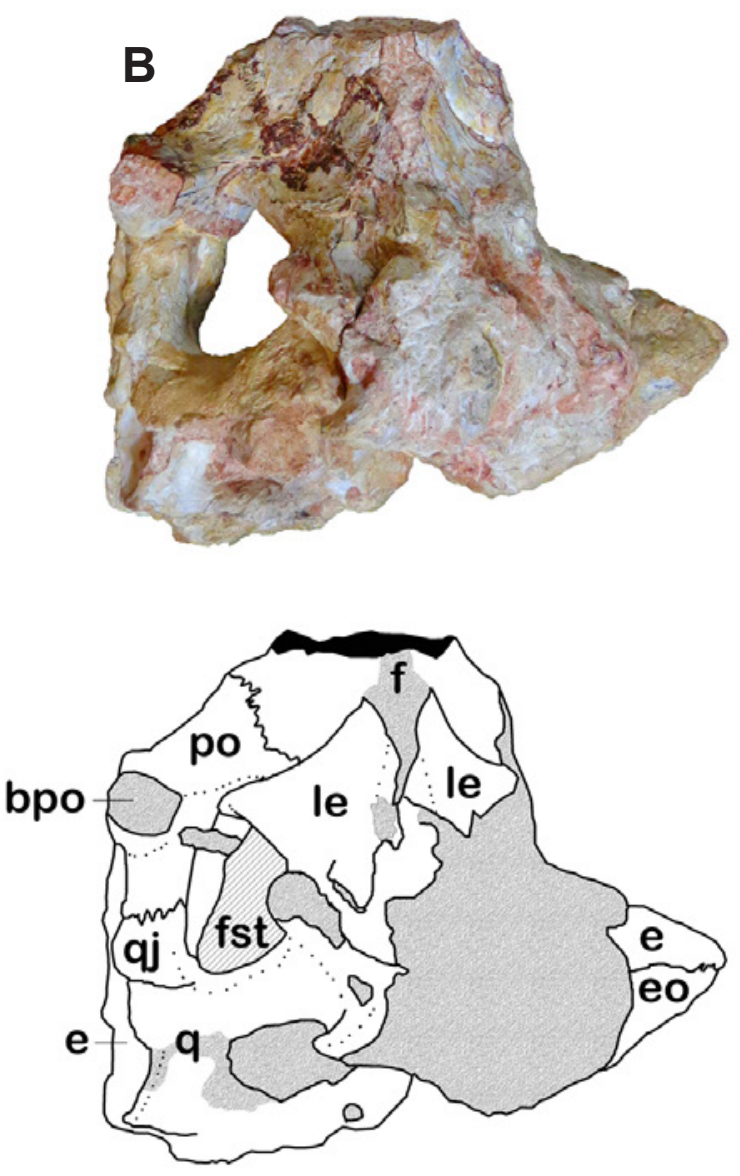

D
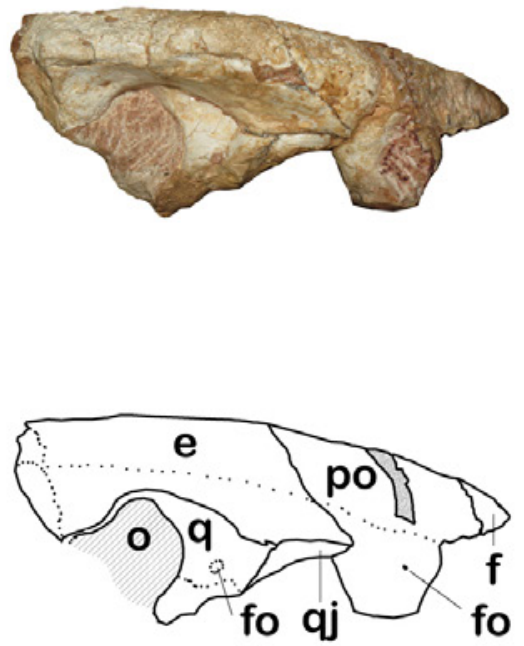

Figura 4. Fóssil MPMA 02-0005/87 em vista dorsal (A), ventral (B), posterior (C) e lateral direita (D). Abreviações: bpo, barra pós-orbital; e, esquamosal; eo, exoccipital; f, frontal; fo, forame; fst, fenestra supratemporal; o, abertura do ouvido; p, parietal; po, pós-orbital, q; quadrado, qj; quadrado-jugal, so; supraoccipital. Escala $=5 \mathrm{~cm}$

Figure 4. Fossil MPMA 02-0005/87 in dorsal (A), ventral (B), posterior (C) and right side (D) views. Abbreviations: bpo, postorbital bar; e, squamosal; eo, exoccipital; f, frontal; fo, foramen; fst, supratemporal fenestra; o, ear opening; p, parietal; po, postorbital; q, quadrate; qj, quadratojugal; so, supraoccipital. Scale bar $=5 \mathrm{~cm}$. 
por rios entrelaçados, eólicos retrabalhados, carbonatos pedogênicos e paleossolos.

A associação fossilífera da Formação Marília em Monte Alto é constituída por saurópodes, terópodes, crocodilifomes e biválvios oriundos de quatro sítios paleontológicos (Roxo, 1929; Ragonha \& Mezzalira, 1985; Santucci \& Bertini, 2001; Bertini et al., 2001; Ghilard et al., 2011; Mendez et al., 2014). O sítio paleontológico de Água Limpa ( $21^{\circ} 16^{\prime} 28.9^{\prime \prime} \mathrm{S}$, $48^{\circ} 32^{\prime} 13.4^{\prime \prime}$ ) localiza-se cerca de $3,5 \mathrm{~km}$ da cidade, está a uma altitude de cerca de $760 \mathrm{~m}$ e é cortado pela estrada municipal que dá acesso ao bairro rural de Água Limpa. É um conjunto de afloramentos constituídos de arenitos esbranquiçados finos a médios, carbonáticos, com frequentes ocorrências de calcretes. Deste ponto, Ragonha \& Mezzalira (1985) apontam a ocorrência de conchas de biválvios atribuídas aos gêneros Iridina e Anodontites, no entanto, os espécimes de crocodiliformes aqui estudados são os únicos fósseis de vertebrados encontrados até o momento.

\section{MATERIAL E MÉTODOS}

Neste estudo são analisados dois espécimes de crocodiliformes descobertos em um mesmo ponto no sítio paleontológico de Água Limpa (Figura 1, Sítio 1), um representado por uma fotografia e outro por um fragmento craniano, depositado no Museu de Paleontologia "Prof. Antonio Celso de Arruda Campos", Monte Alto, Estado de São Paulo, Brasil (MPMA 02-0005/87).

A fotografia consiste no registro feito em 1950 por Isidoro Nunes. A impressão foi feita em papel fotográfico da marca LEONAR, a imagem ocupa uma área de $53 \mathrm{~mm}$ X $53 \mathrm{~mm}$ e tem uma margem branca de $5 \mathrm{~mm}$. Está retratada a parte anterior do rostro de um crocodiliforme em vista lateral esquerda ainda no afloramento, além da mão e antebraço do servidor José Pierre (Figura 3).

O fóssil MPMA 02-0005/87 descoberto em 1987, consiste de um fragmento do teto crânio (Figura 4) apresenta algumas quebras e deslocamento de alguns ossos, que inviabilizam a remoção da matriz de algumas regiões do fóssil. O lado direito está mais bem preservado e observam-se, integral ou parcialmente, os ossos que margeiam a fenestra supratemporal (frontal, pós-orbital, esquamosal e parietal), além do quadradojugal, quadrado e látero-esfenoide; do lado esquerdo foi possível identificar os ossos pós-orbital, esquamosal, supraoccipital, exoccipital e látero-esfenoide.

A preparação do fóssil foi feita de forma mecânica, sendo removido da matriz com a utilização de martelo pena e pequenos cinzeis. A peça foi consolidada com a aplicação de solução de cola branca e água. Para análise da fotografia de 1950 foi feita a digitalização da mesma em alta resolução (300 pixels/polegada) e utilizou-se o software Photoshop CS6, para facilitar a visualização de estruturas, através de utilização do zoom e de filtros de nitidez. Foi possível evidenciar algumas estruturas prémaxilares, maxilares e mandibulares. Os dois espécimes estudados foram comparados prioritariamente com os notossúquios avançados, Baurusuchidae, Peirosauridae, e Trematochampsidae, por representarem os grupos encontrados na Bacia Bauru.

\section{RESULTADOS}

\section{Análise do espécime fotografado nos anos 1950}

A fotografia foi interpretada como sendo o rostro de um crocodiliforme de grande porte em vista lateral esquerda (Figura 3). Algumas estruturas estão bem evidentes, principalmente na região da maxila, onde é possível identificar os dentes de maneira segura. Usando a mão flexionada da imagem como escala e estimando o seu comprimento entre 150 e $200 \mathrm{~mm}$, a parte do fóssil representada na imagem teria entre 300 e $400 \mathrm{~mm}$ de comprimento e na região mais alta entre 135 e $180 \mathrm{~mm}$ de altura. O rostro é longo, a margem ventral da pré-maxila tem um contorno curvo, com a convexidade voltada para baixo. A região que se presume ser o contato com o maxilar apresenta uma reentrância dorsal e a porção do maxilar posterior a junção pré-maxilar-maxilar também apresenta o contorno ventral similar ao do pré-maxilar. É possível identificar apenas quatro dentes no pré-maxilar, dois dentes posteriores e dois anteriores (quase na margem anterior da pré-maxila), sendo que todos eles não apresentam os ápices das coroas preservados. O primeiro da segunda série é hipertrofiado. Um espaço é observado entre estas duas séries, podendo tratar-se de um diastema ou de uma região onde os dentes não estavam preservados. A sutura com o maxilar não está visível. A região exposta da maxila exibe os cinco primeiros dentes arranjados sobre uma borda curva, convexa para baixo. O primeiro dente exibe apenas a base da coroa. O segundo dente está quebrado longitudinalmente, expondo parte da raiz, a região interna e o contorno da coroa; sua margem posterior é reta. Os três dentes seguintes tiveram somente partes das bases das coroas preservadas e aparentemente o terceiro dente era o maior da série. Cinco foramens neurovasculares maxilares estão bem evidentes, dois entre o primeiro e segundo dentes maxilares e outros três próximos ao terceiro dente. Uma pequena porção da mandíbula está visível, na região de contato prémaxila-maxila. Observa-se o molde interno de um dente aparentemente hipertrofiado com parte da coroa, seguido de um outro dente menor adentrando na rocha.

\section{Descrição do fóssil MPMA 02-0005/87}

Ornamentação: a superfície dorsal do crânio está levemente desgastada, mas há indícios da presença de pequenas perfurações circulares nas superfícies dorsais do frontal, pós-orbital e esquamosal. Aberturas cranianas: a fenestra supratemporal direita apresenta a margem externa quase que integralmente preservada; tem o aspecto circular; a fossa apresenta as paredes verticais, salvo no contato entre frontal, parietal e pós-orbital, onde ocorre uma discreta exposição dorsal. As margens anterior e dorsal da abertura do ouvido estão preservadas e são margeadas pelo quadrado; a incisura ótica tem um contorno semicircular e na margem dorsal, ocorre uma leve reentrância. Apenas a região mais 
posterior da órbita direita está preservada na região láteroventral do pós-orbital, frontal e látero-esfenoide. Frontal: é um osso largo, sua extremidade mais lateral (em vista dorsal) está quase na mesma linha da margem lateral da fenestra supratemporal. A superfície entre as suturas com os pós-orbitais é marcada por uma suave depressão na porção medial. Contata o pós-orbital póstero-lateralmente através de uma sutura ampla e bem marcada. O contato com o parietal ocorre através de uma sutura levemente curva; o contato na região medial ocorre numa linha pouco à frente do início das exposições dorsais das fossas supratemporais. Os processos látero-posteriores adentram essas fossas e contatam médioposteriormente os parietais e lateralmente os pós-orbitais. Em vista ventral, observam-se os contatos látero-dorsal com o pós-orbital e médio-ventral com os látero-esfenoides. Pósorbital: é um osso bem robusto; a porção mais anterior que compõe o teto craniano e a parte ventral da barra pós-orbital não estão preservadas. Compõe parte da margem anterior e lateral da fenestra supratemporal, onde tem um aspecto cilíndrico; junto com o quadrado-jugal compõe o ápice da fenestra infratemporal. Contata o esquamosal pósteroventralmente através de uma sutura, que em vista lateral, é oblíqua em relação a plano dorsal. A porção dorsal da barra pós-orbital tem aspecto cilíndrico, e é marcada pela presença de um forâmen na sua lateral. Parietal: apenas seus limites anteriores estão bem definidos; a porção medial da metade posterior não está preservada, porém uma superfície mais lateral está preservada e volta-se médio-dorsalmente. Uma parte do crânio está quebrada e deslocada lateralmente; observa-se grande parte da superfície dorsal do parietal e o seu contato com supraoccipital. Esquamosal: apresenta as superfícies dorsal e lateral ornamentadas e as ventral e láteroventral lisas. Apresenta uma ampla exposição dorsal entre a margem da fenestra supratemporal e a margem posterior do crânio. $\mathrm{O}$ ramo medial bordeja a fenestra supratemporal e contata o parietal; na superfície dorsal, o contato esquamosalparietal é direcionado póstero-medialmente e dentro da fossa supratemporal, é ventral e ventro-medial em relação ao parietal. Possui uma projeção anterior cuneiforme (vista lateralmente) que contata ântero-dorsalmente o pós-orbital, e ventralmente o quadrado-jugal. Na superfície lateral externa, posterior ao pós-orbital, está bem evidente o sulco para a fixação da tampa auricular superior, que se torna menos raso na linha da margem anterior da abertura auricular. Próximo à abertura do ouvido, o osso contata o quadrado ventral $\mathrm{e}$ médio-ventralmente. Os limites posteriores do esquamosal não estão claros. Quadrado: está preservada a porção mais dorsal do osso. Contata ântero-ventralmente o quadradojugal, ântero-dorsalmente e dorsalmente o esquamosal, além de compor as margens dorsal e ântero-dorsal da abertura ótica. Está presente um forâmen subtimpânico pequeno, compreendido entre a margem do ouvido e a sutura com o quadrado-jugal. Látero-esfenoide: seus processos dorsais são robustos. Dentro da órbita ocular está exposto numa superfície voltada látero-ventralmente e contata o frontal e pequena parte do pós-orbital látero-dorsalmente. Uma pequena projeção cuneiforme contata lateralmente a porção dorsal da barra pós-orbital. Contata o parietal póstero-dorsalmente; uma sutura reta entre tais ossos é observada nas paredes ântero-medial e medial da fenestra supratemporal. Supraoccipital: uma pequena parte do osso esquerdo é notado em vista dorsal; contata o parietal láteroanteriormente. Em vista posterior observa-se uma ampla superfície do osso e o contato com o exoccipital.

\section{DISCUSSÃO E CONCLUSÃO}

As primeiras coletas no Município de Monte Alto foram realizadas no ano de 1918. Tais fósseis foram coletados para o Serviço Geológico e classificados como restos de dinossauros (Mezzalira, 1974). Desconsiderando a citação de MoraesRego (1935), a foto apresentada neste estudo é o registro mais antigo de Crocodyliformes na região. A descoberta e a fotografia do espécime ocorreram na década de 1950, cerca de 20 anos antes da descoberta do espécime de Stratiotosuchus por Rafael G. M. Neto e de aproximadamente 40 anos antes da primeira publicação da ocorrência do grupo na região.

A análise do registro fotográfico revela várias características do espécime, contudo baseia-se em características gerais e o levantamento de dados mais acurados não é possível, assim como uma associação taxonômica segura. A imagem mostra a região anterior de um rostro de um espécime de grande porte; estão evidentes a grande reentrância na margem ventral do rostro (entre pré-maxila e maxila) que abriga o dente hipertrofiado da mandíbula, além da primeira série de dentes maxilares arranjado sobre a margem ventral convexa da maxila. Tais feições são comuns em crocodiliformes essencialmente predadores, como ocorre em várias formas atuais e dentre diversos táxons cretácicos, tais como: Baurusuchus, Stratiotosuchus, Uberabasuchus e Hamadasuchus (Iordansky, 1973; Price, 1945; Campos et al., 2001; Carvalho et al., 2004; 2005; Larsson \& Sues, 2007). A região preservada do rostro (da extremidade anterior até a altura do quinto dente maxilar) é relativamente mais baixa que em baurussuquídeos. A base da coroa do quinto dente maxilar está bem mais alta que o seu antecessor, ao passo que na maioria dos baurussúquídeos o quarto e o quinto dentes maxilares estão implantados quase na mesma altura. Um dente hipertrofiado está presente; possivelmente se trata do penúltimo dente pré-maxilar. Em aspecto geral, a região exposta do exemplar fotografado, assemelha-se muito à observada em a Hamadasuchus e parece sugerir uma segunda onda de dentes maxilares.

O espécime fotografado foi descoberto em arenitos da Formação Marília no sítio paleontológico de "Água Limpa". Deste mesmo contexto é oriundo o fóssil MPMA 02-0005/87, portanto a hipótese de ambos espécimes pertencerem a um mesmo táxon não deve ser descartada. O fóssil MPMA 020005/87 também é de um espécime de grande porte, cuja largura do teto craniano na região das fenestras supratemporais é de $170 \mathrm{~mm}$. Hamadasuchus (ROM 52620), por exemplo, possui o comprimento craniano de $325 \mathrm{~mm}$ e o teto craniano possui $74 \mathrm{~mm}$ de largura (Larsson \& Sues, 2007). 
O fóssil MPMA 02-0005/87 consiste de uma pequena parte do crânio, contudo foi possível comparar com outros táxons da Bacia Bauru. A superfície externa do teto, embora levemente desgastada, exibe sinais de sua ornamentação, constituída de perfurações circulares, como ocorre em peirossaurídeos e trematochampsídeos. Por outro lado, não estão presentes ornamentações conspícuas (como a crista sagital do frontal e as bordas do parietal que margeiam as fenestras supra-temporais) comuns em baurussuquídeos e notossúquios avançados (Carvalho et al., 2004; Zaher et al., 2006; Montefeltro et al., 2011; Iori et al., 2013 Godoy et al., 2014). Os Baurusuchidae possuem fenestra supratemporal subtriangular e com uma ampla fossa que a margeia, principalmente na margem posterior, enquanto no fóssil MPMA 02-0005/87 apenas uma pequena inclinação é observada anteriormente. A exposição dorsal da superfície ornamentada do esquamosal posterior à fenestra é ampla no fóssil MPMA 02-0005/87, enquanto na maioria dos baurussuquídeos se restringe a uma porção bastante estreita, salvo em Aplestosuchus, em que tal região é bem desenvolvida, contudo neste caso a fenestra supratemporal é menor. O contorno da fenestra supra-temporal no fóssil MPMA 02-0005/87 é quase circular, porém não tão simétrica como ocorre em Barreirosuchus (Carvalho et al., 2004, 2005, 2007, 2011; Riff \& Kellner, 2011; Montefeltro et al., 2011; Iori \& Garcia, 2012, Godoy et al., 2014; Pol et al., 2014). O fóssil MPMA 02-0005/87 apresenta um pequeno forâmen subtimpânico preservado, porém é impossível afirmar se este seria único, pois a região periótica não está completa. Segundo Montefeltro et al. (2016), tal estrutura pode estar ausente em alguns táxons (e.g. Pepesuchus) e variar de número em outros, com uma complexidade maior nos baurussuquídeos e notossúquios avançados.

Os poucos caracteres observados no fóssil MPMA 020005/87 são distintos dos ocorridos em Baurusuchidae; o padrão de ornamentação do espécime em análise sugere uma maior afinidade com Peirosauridae ou Trematochampsidae, famílias também presentes na Formação Marília do Triângulo Mineiro (Price, 1955; Carvalho et al., 2004).

Embora a Bacia Bauru venha suprindo muita informação acerca de Crocodyliformes, a grande maioria dos espécimes foi descoberta em depósitos da Formação Adamantina. Dentre os táxons da Formação Marília, quatro gêneros são conhecidos: Peirosaurus, Itasuchus, Uberabasuchus e Labidiosuchus, todos da região de Uberaba, MG (Price, 1955; Carvalho et al., 2004; Kellner et al., 2011). Os espécimes apresentados neste estudo representam os primeiros fósseis figurados da Formação Marília no Estado de São Paulo, e uma das raras ocorrências de crocodiliformes no Membro Echaporã.

O registro de paleovertebrados da Formação Marília da região de Monte Alto estava restrita aos titanossaurídeos e abelissauroides (Bertini et al., 2001; Mendez et al., 2014). Os crocodiliformes de grande porte do sítio de Água Limpa passam a compor esse cenário, e juntos com os terópodes passam a figurar dentre os grandes predadores do Maastrichtiano da região de Monte Alto.

\section{AGRADECIMENTOS}

Gostaríamos de agradecer a I. Nunes (in memoriam) pela execução do registro fotográfico e pela doação da fotografia, a J. Pierre (in memoriam) pela descoberta do campo paleontológico, e a todos os colaboradores envolvidos nas pesquisas. Agradecemos aos revisores, um deles F.C. Montefeltro, e à editora A.M. Ribeiro pelos valiosos comentários nas versões anteriores deste manuscrito.

\section{REFERÊNCIAS}

Andrade, M.B. \& Bertini, R.J. 2008. A new Sphagesaurus (Mesoeucrocodylia: Notosuchia) from the Upper Cretaceous of Monte Alto City (Bauru Basin, Brazil), and a revision of the Sphagesauridae. Historical Biology, 20:101-136. doi:10.1080/08912960701642949

Batezelli, A. 2015. Continental systems tracts of the Brazilian Cretaceous Bauru Basin and their relationship with the tectonic and climatic evolution of South America. Basin Research. doi:10.1111/bre.12128

Bertini, R.J.; Santucci, R.M. \& Arruda-Campos, A.C. 2001. Titanossauros (Sauropoda: Saurischia) no Cretáceo Superior continental (Formação Marília, Membro Echaporã) de Monte Alto, estado de São Paulo, e correlação com formas associadas do Triângulo Mineiro. Geociências, 20:93-103.

Campos, D.A.; Suarez, J.M.; Riff, D. \& Kellner, A.W.A. 2001. Short note on a new Baurusuchidae (Crocodyliformes, Metasuchia) from the Upper Cretaceous of Brazil. Boletim do Museu Nacional, Geologia, 57:1-7.

Carvalho, I.S.; Campos, A.C.A. \& Nobre, P.H. 2005. Baurusuchus salgadoensis, a new Crocodylomorpha from the Bauru Basin (Cretaceous), Brazil. Gondwana Research, 8:11-30. doi:10.1016/S1342-937X(05)70259-8

Carvalho, I.S.; Ribeiro, L.C.B. \& Avilla, L.S. 2004. Uberabasuchus terrificus sp. nov, a new Crocodylomorpha from the Bauru Basin (Upper Cretaceous), Brazil. Gondwana Research, 7:975-1002. doi:10.1016/S1342-937X(05)71079-0

Carvalho, I.S.; Teixeira, V.P.A.; Ferraz, M.L.F.; Ribeiro, L.C.B.; Martinelli, A.G.; Neto, F.M.; Sertich, J.J.W.; Cunha, G.C.; Cunha, I.C. \& Ferraz, P.F. 2011. Campinasuchus dinizi gen. et sp. nov., a new Late Cretaceous baurusuchid (Crocodyliformes) from the Bauru Basin, Brazil. Zootaxa, 2871:19-42.

Carvalho, I.S.; Vasconcellos, F.M. \& Tavares, S.A.S. 2007. Montealtosuchus arrudacamposi, a new peirosaurid crocodile (Mesoeucrocodylia) from the Late Cretaceous Adamantina Formation of Brazil. Zootaxa, 1607:35-46.

Dal' Bó, P.F.F; Basilici, G.; Angelica, R.S. \& Ladeira, F.S.B. 2009. Paleoclimatic interpretations from pedogenic calcretes in a Maastrichtian semi-arid eolian sand-sheet palaeoenvironment: Marília Formation (Bauru Basin, southeastern Brazil). Cretaceous Research, 30:659-675. doi:10.1016/j.cretres.2008.12.006

Dias-Brito, D.; Musacchio, E.A.; Castro, J.C.; Maranhão, M.S.A.S.; Suarez, J.M. \& Rodrigues, R. 2001. Grupo Bauru: uma unidade continental do Cretáceo no Brasil - concepções baseadas em dados micropaleonotológicos, isotópicos e estratigráficos. Rèvue Paléobiologie, 20:245-304.

Fachini, T.S. \& Iori, F.V. 2009. Primeiro registro de Squamata na região do município de Monte Alto, estado de São Paulo (Bacia Bauru, Cretáceo Superior). In: CONGRESSO BRASILEIRO DE PALEONTOLOGIA, 21, 2009. Livro de Resumos, Belém, p. 172. 
Fernandes, L.A. 2004. Mapa Litoestratigráfico da Parte Oriental da Bacia Bauru (PR, SP, MG). Boletim Paranaense de Geociências, 55:53-66.

Fernandes, L.A. \& Coimbra, A.M.A. 1996. Bacia Bauru (Cretáceo Superior, Brasil). Anais da Academia Brasileira de Ciências, 68:195-205.

Ghilardi, R.P.; D’Agosta, F.C.P.; Alves, K. \& Arruda-Campos, A.C. 2011. Tafononia de moluscos fósseis do Grupo Bauru (Cretáceo Superior, Bacia Bauru) na região do município de Monte Alto, São Paulo, Brasil. Boletim do Museu Paraense Emílio Goeldi, 6:197-206.

Godoy, P.L.; Montefeltro, F.C.; Norell, M.A. \& Langer, M.C. 2014. An Additional Baurusuchid from the Cretaceous of Brazil with Evidence of Interspecific Predation among Crocodyliformes. PLoS ONE, 9:e97138. doi:10.1371/journal.pone.0097138

Iordanski, N.N. 1973. The skull of the Crocodylia. In: C. Gans \& T.S. Parsons (eds.) Biology of the Reptilia, Academic Press, p. 201-262.

Iori, F.V.; Arruda-Campos, A.C. \& Carvalho, I.S. 2009a. Ocorrência de um Itassuquídeo de grande porte no Município de Monte Alto, Estado de São Paulo (Bacia Bauru, Cretáceo Superior). In: CONGRESSO BRASILEIRO DE HERPETOLOGIA, 4, 2009. Anais, Pirenópolis, p. 1.

Iori, F.V. \& Carvalho, I.S. 2009. Morrinhosuchus luziae, um novo Crocodylomorpha Notosuchia da Bacia Bauru, Brasil. Revista Brasileira de Geociências, 39:717-725.

Iori, F.V. \& Carvalho, I.S. 2011. Caipirasuchus paulistanus, a new sphagesaurid (Crocodylomorpha, Mesoeucrocodylia) from the Adamantina Formation (Upper Cretaceous, Turonian-Santonian), Bauru Basin, Brazil. Journal of Vertebrate Paleontology, 31:1255-1264. doi:10.1080/02724634.2011.602777

Iori, F.V.; Carvalho, I.S. \& Arruda-Campos, A.C. 2009b. Os Crocodylomorpha da Formação Marília no Município de Monte Alto, Estado de São Paulo (Bacia Bauru, Cretáceo Superior). In: CONGRESSO BRASILEIRO DE PALEONTOLOGIA, 21, 2009. Livro de Resumos, Belém, p. 191.

Iori, F.V.; Carvalho, I.S. \& Marinho, T.S. 2016. Postcranial skeletons of Caipirasuchus (Crocodyliformes, Notosuchia, Sphagesauridae) from the Upper Cretaceous (TuronianSantonian) of the Bauru Basin, Brazil. Cretaceous Research, 60:109-120. doi: 10.1016/j.cretres.2015.11.017

Iori, F.V. \& Garcia, K.L. 2012. Barreirosuchus franciscoi, um novo Crocodylomorpha Trematochampsidae da Bacia Bauru, Brasil. Revista Brasileira de Geociências, 42:397-410. doi:10.5327/ Z0375-75362012000200013

Iori, F.V.; Marinho, T.S.; Carvalho, I.S. \& Arruda-Campos, A.C. 2013. Taxonomic reappraisal of the sphagesaurid crocodyliform Sphagesaurus montealtensis from the Late Cretaceous Adamantina Formation of São Paulo state, Brazil. Zootaxa, 3686:183-200. doi:10.11646/zootaxa.3686.2.4

Iori, F.V.; Marinho, T.S.; Carvalho, I.S. \& Frare, L.A.S. 2015. Análise da morfologia craniana de um novo espécime de Morrinhosuchus luziae (Crocodyliformes, Notosuchia) da região de Monte Alto - SP (Bacia Bauru, Cretáceo Superior). In: CONGRESSO BRASILEIRO DE PALEONTOLOGIA, 24, 2015. Boletim de resumos, Crato, p. 184.

Kellner, A.W.A.; Figueiredo, R.G.; Azevedo, S.A.K. \& Campos, D.A. 2011. A new cretaceous notosuchian (Mesoeucrocodylia) with bizarre dentition from Brazil. Zoological Journal of the Linnean Society, 163:S115. doi:10.1111/j.1096-3642.2011.00711.x

Larsson H.C.E. \& Sues H.D. 2007. Cranial osteology and phylogenetic relationships of Hamadasuchus rebouli (Crocodyliformes: Mesoeucrocodylia) from the Cretaceous of Morocco. Zoological Journal of the Linnean Society, 149:533-567. doi:10.1111/ j.1096-3642.2007.00271.x
Méndez, A.H.; Novas, F.E. \& Iori, F.V. 2014. New record of Abelisauroid Theropods from the Bauru Group (Upper Cretaceous), São Paulo State, Brazil. Revista Brasileira de Paleontologia, 17:23-32. doi:10.4072/rbp.2014.1.03

Mezzalira, S. 1974. Contribuição ao conhecimento da estratigrafia e paleontologia do Arenito Bauru. Boletim do Instituto Geográfico e Geológico, 51:1-163.

Montefeltro, C.M.; Andrade, D.V. \& Larsson, H.C.C. 2016. The evolution of the meatal chamber in crocodyliforms. Journal of Anatomy, 228:838-863. doi:10.1111/joa.12439

Montefeltro, F.C.; Larsson, H.C.E. \& Langer, M.C. 2011. A new baurusuchid (Crocodyliformes, Mesoeucrocodylia) from the Late Cretaceous of Brazil and the phylogeny of Baurusuchidae. PLoS ONE, 6: e21916. doi:10.1371/journal.pone.0021916

Moraes Rego, L.F. 1935. Camadas cretáceas do sul do Brasil. Anais da Escola Politécnica, 4:231-274.

Paula e Silva, F.; Chang, H.K. \& Caetano-Chang, M.R. 2003. Perfis de referência do Grupo Bauru (K) no Estado de São Paulo. Revista Brasileira Geociências, 22:21-32.

Pinheiro, A.E.P.; Bertini, J.R.; Andrade, M.B. \& Neto, M.B.M. 2008. A new specimen of Stratiotosuchus maxhechti (Baurusuchidae, Crocodyliformes) from the Adamantina Formation (Upper Cretaceous) southeastern Brazil. Revista Brasileira de Paleontologia, 11:37-50. doi:10.4072/rbp.2008.1.04

Pol, D.; Nascimento, P.M.; Carvalho, A.B.; Riccomini, C.; PiresDomingues, R.A. \& Zaher, H. 2014. A new notosuchian from the Late Cretaceous of Brazil and the phylogeny of advanced notosuchians. PLoS ONE 9:e93105. doi:10.1371/journal. pone.0093105

Price, L.I. 1945. A new reptile from the Late Cretaceous of Brazil. Serviço Geológico Mineralogia, 25:1-8.

Price, L.I. 1950. On a new Crocodilia, Sphagesaurus from the Cretaceous of State of São Paulo, Brazil. Anais da Academia Brasileira de Ciências, 22:77-83.

Price, L.I. 1955. Novos crocodilídeos dos arenitos da Série Bauru, Cretáceo do estado de Minas Gerais. Anais da Academia Brasileira de Ciências, 27:487-498.

Ragonha, E.W. \& Mezzalira, S. 1985. Nova malacofauna dulciaquícola no grupo Bauru (K Sup.) de Monte Alto (SP),Brasil. In: CONGRESSO BRASILEIRO DE PALEONTOLOGIA, 9 , 1985. Boletim de Resumos, Fortaleza, p. 70.

Riff, D. \& Kellner, A.W.A. 2011. Baurusuchid crocodyliforms as theropod mimics: clues from the skull and appendicular morphology of Stratiotosuchus maxhechti (Upper Cretaceous of Brazil) The Linnean Society of London, Zoological Journal of the Linnean Society, 163:S37-S56. doi:10.1111/j.10963642.2011.00713.x

Roxo, M.G.O. 1929. Geologia da região entre o Rio do Peixe e o Paranapanema. Rio de Janeiro, Serviço Geológico e Mineralógico, p. 35-40. (Relatório Anual do Diretor).

Santucci, R.M. \& Arruda-Campos, A.C. 2011. A new sauropod (Macronaria, Titanosauria) from the Adamantina Formation, Bauru Group, Upper Cretaceous of Brazil and the phylogenetic relationships of Aeolosaurini. Zootaxa, 3085:1-33.

Santucci R.M. \& Bertini, R.J. 2001. Distribuição paleogeográfica e biocronológica dos titanossauros (Saurischia, Sauropoda) do Grupo Bauru, Cretáceo Superior do Sudeste Brasileiro. Revista Brasileira de Geociências, 31:307-314.

Tavares, S.A.S.; Ricardi-Branco, F.; Iori, F.V.; Tavares, P.G.R. \& Arruda-Campos, A.C. 2010. Dentes isolados de crocodilomorfos da Fazenda Santa Irene, Município de Monte Alto, SP, Brasil. (Bacia Bauru - Formação Adamantina). In: CONGRESSO 
BRASILEIRO DE PALEONTOLOGIA DE VERTEBRADOS, 7, 2010. Boletim de resumos, Rio de Janeiro, p. 66.

Tavares, S.A.S.; Ricardi-Branco, F. \& Santucci, R.M. 2014. Theropod teeth from the Adamantina Formation (Bauru Group, Upper Cretaceous), Monte Alto, São Paulo, Brazil. Cretaceous Research, 50:59-71. doi:10.1016/j.cretres.2014.03.021
Zaher, H.; Pol, D.; Carvalho, A.B.; Riccomini, C.; Campos, D. \& Nava. W. 2006. Redescription of the cranial morphology of Mariliasuchus amarali, and its phylogenetic affinities (Crocodyliformes, Notosuchia). American Museum Novitates 3512:1-40.

Received in May, 2016; accepted in August, 2016. 